\title{
Machine Learning-Based Classification of Vector Vortex Beams
}

\author{
Taira Giordani, ${ }^{1}$ Alessia Suprano, ${ }^{1}$ Emanuele Polino $\odot,{ }^{1}$ Francesca Acanfora $\odot,{ }^{1}$ Luca Innocenti $\odot,{ }^{2}$ Alessandro Ferraro, ${ }^{2}$ \\ Mauro Paternostro, ${ }^{3}$ Nicolò Spagnolo $\odot,{ }^{1}$ and Fabio Sciarrino ${ }^{1,4}$ \\ ${ }^{1}$ Dipartimento di Fisica, Sapienza Università di Roma, Piazzale Aldo Moro 5, I-00185 Roma, Italy \\ ${ }^{2}$ Centre for Theoretical Atomic, Molecular, and Optical Physics, School of Mathematics and Physics, \\ Queen's University Belfast, BT7 INN Belfast, United Kingdom \\ ${ }^{3}$ Centre for Theoretical Atomic, Molecular, and Optical Physics, School of Mathematics and Physics, \\ Queen's University Belfast, BT7 1NN Belfast, United Kingdom \\ ${ }^{4}$ Consiglio Nazionale delle Ricerche, Istituto dei sistemi Complessi (CNR-ISC), Via dei Taurini 19, 00185 Roma, Italy
}

(Received 26 September 2019; accepted 16 March 2020; published 20 April 2020)

\begin{abstract}
Structured light is attracting significant attention for its diverse applications in both classical and quantum optics. The so-called vector vortex beams display peculiar properties in both contexts due to the nontrivial correlations between optical polarization and orbital angular momentum. Here we demonstrate a new, flexible experimental approach to the classification of vortex vector beams. We first describe a platform for generating arbitrary complex vector vortex beams inspired to photonic quantum walks. We then exploit recent machine learning methods-namely, convolutional neural networks and principal component analysis - to recognize and classify specific polarization patterns. Our study demonstrates the significant advantages resulting from the use of machine learning-based protocols for the construction and characterization of high-dimensional resources for quantum protocols.
\end{abstract}

DOI: 10.1103/PhysRevLett.124.160401

Introduction.-Light is endowed with orbital angular momentum (OAM) [1,2], a degree of freedom associated with structured, nonplane wavefronts, and characterized by an azimuthal phase dependence. When a nontrivial phase dependence is coupled with a helicoidal transverse polarization pattern, one talks of a vector vortex beam (VVB) $[2,3]$. The interest in such states is motivated by the applications in multiple fields of classical and quantum optics [4,5]: from particle trapping to metrological applications in microscopy [6,7], and for OAM-based communications schemes in free-space and in fiber [8,9]. VVBs are also often employed in quantum information protocols due to the hyperentanglement between their polarization and spatial degrees of freedom. Photonic platforms for quantum sensing and metrology leveraging such encoding have also been reported [10,11]. OAM-based schemes for investigating quantum causal structures [12], quantum communication and cryptography [13-18], quantum walks [19-21], quantum simulation [22,23], and quantum state engineering [24,25], have been previously demonstrated.

Despite the potential of VVBs, many questions regarding the decoding of information stored in OAM and polarization remain unanswered. Various techniques of OAM-demultiplexing envisage the need of additional instruments-such as interferometry [26-28] or spatial filtering [29-31] — to be efficiently implemented. These introduce detrimental effects of loss and noise [32]. Moreover, the challenge of performing state tomography in such a high-dimensional framework, a fundamental task in quantum information processing [33,34], can hardly be overestimated. The design and demonstration of reliable techniques for the generation and classification of VVBs is thus highly desirable. Indeed, substantive efforts on finding novel platforms are the subject of intense research activities $[6,7,35,36]$, including in integrated photonics [37-39] and generation by plasmonic metasurfaces $[40,41]$.

Recently, machine learning (ML) has emerged as a versatile toolbox to tackle a variety of tasks arising in experimental platforms. It has proven useful, in particular, to ease the characterization of quantum protocols and dynamics [42-52]. In the context of structured light, neural networks (NNs) have been used to classify OAM states of classical light for long distance free-space communication, even in the presence of environmental turbulence [53-58]. In this Letter, we apply ML to characterize experimental VVBs generated using a platform based on photonic quantum walks (QWs) in the OAM and polarization degrees of freedom [24,25]. Our approach requires neither additional interferometry stabilization nor spatial filtering, thus providing a robust strategy to decode information stored in VVBs, and is therefore a promising pathway towards managing higher-dimensional quantum systems.

We leverage both supervised and unsupervised learning techniques. We start by training a convolutional neural network (CNN) to classify experimental images belonging to predefined classes of states. This method gives good prediction accuracy, while remaining fairly problem-agnostic and thus useful for diverse applications. However, while 
providing high prediction accuracy, NN-based methods are difficult to interpret. We thus also propose an alternative technique based on the joint application of dimensionality reduction (DR) and supervised learning. This method provides a geometrical description of the underlying space associated to the experimental data. While significantly easier to use, such approach gives comparable results to $\mathrm{CNN}$, at the cost of being more tailored to the specifics of the problem.

Our work makes significant steps forward with respect to previous endeavors: while Refs. [53-58] leverage NNs to process OAM states, our work is the first to tackle VVBs. Moreover, owing to the variety of techniques we deploy, we can address both classification and regression tasks, thus enabling the reconstruction of the input states in relevant cases of structured light beams. Our findings demonstrate the reliability of a broader class of ML methods, providing novel recognition methods to deal with VVB, which are a building block for several information protocols with highdimensional systems.

Experimental generation of vector vortex beams.OAM-endowed states of light can be described using Laguerre-Gauss (LG) modes. These are solutions of the Helmholtz equation in the paraxial approximation, indexed by two integer numbers $(m, p)$, the former describing the azimuthal phase structure of the beam, and the latter describing its radial intensity profile. Each LG mode carries a set amount of angular momentum, which in the singlephoton regime equals $\hbar m$ [1]. VVBs can be obtained by superposing orthogonal polarizations to LG modes [2]. More specifically, the electric field $\vec{E}_{m_{1} m_{2} p}$ of a VVB decomposes as the sum of two LG modes with the same $p$ and different azimuthal numbers $m_{1}>m_{2}$ carried by orthogonal polarizations: $\vec{E}_{m_{1} m_{2} p}=\vec{e}_{L} \cos (\theta / 2) \mathrm{LG}_{m_{1} p}+$ $\vec{e}_{R} e^{i \phi} \sin (\theta / 2) \mathrm{LG}_{m_{2} p}$, where $\theta \in[0, \pi], \phi \in[0,2 \pi]$ and the unit vectors $\vec{e}_{L, R}$ stand for left and right circular polarization, respectively. For the purpose of this work we can ignore the radial number, setting $p=0$. For any given value of the parameters $\left(m_{1}, m_{2}, \theta, \phi\right)$, the polarization pattern of a VVB can be mapped onto a generalized Poincaré sphere (cf. Fig. 1). In particular, we use the higher-order Poincaré representation in which the poles represent eigenstates of the total angular momentum but with opposite signs [59]. These polarization patterns are reconstructed via the Stokes parameters $S_{j}(j=1,2,3)$, obtained by measuring the output intensities $I_{b_{j}, 1}, I_{b_{j}, 2}$ associated with a given choice of polarization basis $\left\{b_{j}\right\}=\left\{b_{1}=(H, V), b_{2}=\right.$ $\left.(D, A), b_{3}=(L, R)\right\}$ as $S_{b_{j}}=\left(I_{b_{j}, 1}-I_{b_{j}, 2}\right) /\left(I_{b_{j}, 1}+I_{b_{j}, 2}\right)$. For a VVB, the values of $S_{j}$ depend on the coordinates in the transverse propagation plane [60]. To visualize the polarization patterns of VVBs, we use an RGB color encoding in which the values of $S_{j}$ are interpreted as strengths of the corresponding color. In Figs. 1(b) and 1(c) we report an example of such a color map for radially polarized

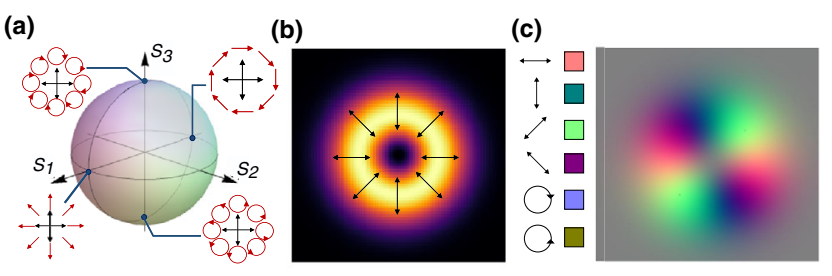

(d)

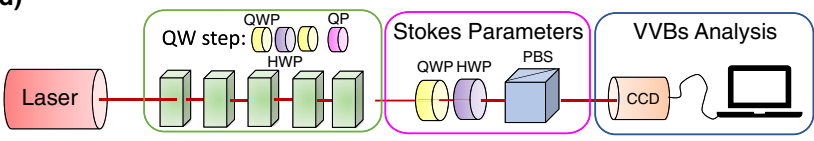

FIG. 1. (a) Higher-order Poincaré sphere representation for $\left|m_{1,2}\right|=1$. Each point on the sphere surface corresponds to specific polarization patterns. (b) A radially polarized VVB: at a given point in the transverse plane the polarization vector has a different orientation. The Stokes parameters vary accordingly in the plane. (c) Color encoding of the polarization pattern. The legend reports the correspondence between colors and the various polarizations. On the right we have the resulting color pattern for the VVB in panel (b). Gray color corresponds to unpolarized light. (d) Experimental apparatus for the generation of VVBs. A continuous-wave laser emits a Gaussian beam TEM $_{00}$ at $808 \mathrm{~nm}$. Light undergoes a 5-step quantum walk realized through a sequence of wave plates and $q$ plates. A CCD camera-based detection stage acquires information on the Stokes parameters and the polarization pattern. Based on the intensity measured at each pixel of the camera, Stokes parameters are evaluated and converted into RGB-colored pictures.

VVBs. A natural way to generate VVBs is using $q$ plates $[60,61]$, which are inhomogenous birefringent plates modifying the OAM of the incoming light conditionally to its polarization. In our scheme, VVBs are generated via a sequence of polarization-controlling wave plates interspersing 5 cascaded $q$ plates [cf. Fig. 1(d)]. The apparatus implements a discrete-time QW in the angular momentum, where the order of LG modes takes the role of the walker and it is changed according to the polarization state, which embodies the coin degree of freedom [19-21,24,25]. This allows us to generate several classes of VVBs with OAM quantum numbers taking odd values in the interval $\{-5, \ldots, 5\}$. We then collect images associated with different VVBs and use them to train and benchmark our ML-based approaches to classification, as discussed in the next sections.

Classification via convolutional neural networks.-We show here how to train a $\mathrm{CNN}$ to retrieve the parameters $\left(m_{1}, m_{2}\right)$ characterizing a given VVB from experimentally measured Stokes parameters. CNNs are translation-invariant deep NNs well-suited for image classification [62], to recognize off-center images and segmented handwritten digits $[63,64]$, and for facial recognition tasks [65]. In their simplest form, CNNs work by first applying a convolutional layer, which consists of a series of nonlinear transformations applied to the input images, followed by a maxpooling layer, which downsamples and filters the information extracted by the previous layer. Finally, a fully 


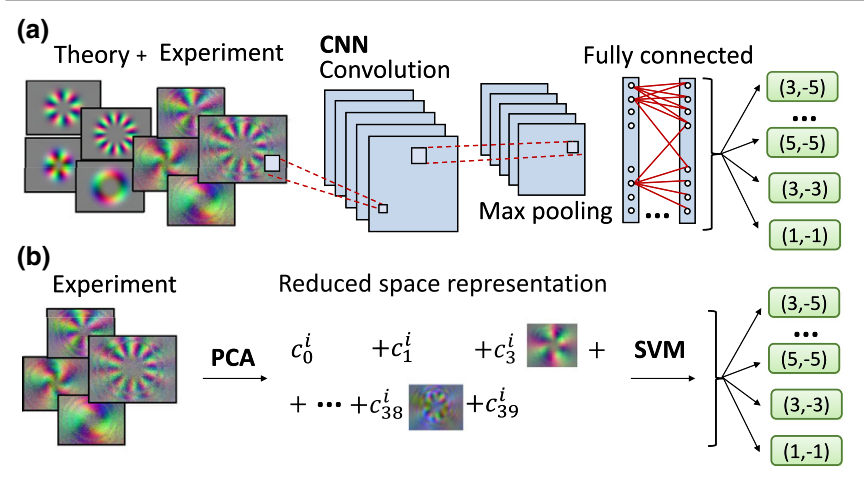

FIG. 2. (a) Schematic representation of VVBs classification via CNNs. (b) Classification scheme using linear PCA. After reducing the dimensionality of the dataset via PCA, a linear SVM is used to classify experimental images.

connected layer operates as a classifier, categorizing the information extracted in the previous layers into one of a small number of possible output categories (cf. Refs. [66-70] and Fig. 2).

The network is first fed with a training set made out of simulated images of VVBs achievable with a five-step QW. The task is then to discern between 15 classes, corresponding to the pairs $\left(m_{1}, m_{2}\right)$ in Fig. 3(a). For each class we generate states with $\theta=\pi / 2$ and $\phi \in[0,2 \pi]$. The size of the training set is 400 images per class. Additional 100 simulated images per class are used to benchmark the performance during training. In these conditions, the network achieves an accuracy of $100 \%$. The term accuracy is used here to refer to the fraction of correctly classified images. We then collect 100 experimental images per class, to use as a new validation set [cf. Fig. 2(a)]. Figures 3(a)-3(b) show the average accuracy per class against the fraction of experimental images added to the training set. The addition of a small fraction of experimental images to the training set improves the capability of the network to take into account deviations of the experimental states from ideal LG modes [71,72, 78-80] [cf. Fig. 3(b)]. An average accuracy of $\sim 0.989$ is already obtained when $12.5 \%$ of the training set is composed of experimental images. To further highlight the performance of the network, we also trained a CNN using exclusively experimental images, but using a small number of images in the training phase. Using only 20 images per class, we already get an accuracy of 0.99 to classify the rest of the experimental images (which are 1668 in total).

We use a similar approach to retrieve the position on the Poincaré sphere corresponding to states generated with fixed $\left(m_{1}, m_{2}\right)$. In particular, we test the performance of CNNs to retrieve the values $(\theta, \phi)$ of VVBs corresponding to $m_{2}=-m_{1}=1$. The $\mathrm{CNN}$ is thus trained to discriminate both rotations in the polarization patterns (corresponding to changes of $\phi$ ), and variations in the color tone (corresponding to changes of $\theta$ ). To frame this as a classification task,
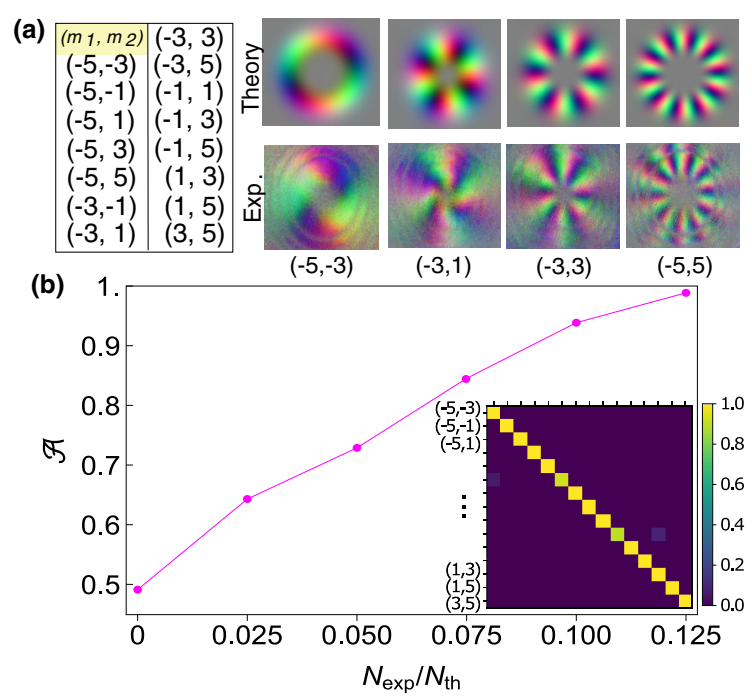

FIG. 3. (a) Simulated and experimental images of VVBs corresponding to some of the values $\left(m_{1}, m_{2}\right)$ given in the table. (b) Scaling of the average accuracy $\mathcal{A}$ when classifying states into one of the $15 \mathrm{VVB}$ classes, against the fraction of experimental images added to the training set. The leftmost point refers to the case in which only simulated images are used to train the network. Inset: truth table reporting how the network classifies images belonging to each class. Each row (column) corresponds to a possible pair $\left(m_{1}, m_{2}\right)$. The matrix elements have been averaged over 100 experimental images per class.

we partition the sphere in 26 disjoint sectors. Working in spherical coordinates, we partition $\theta$ in 3 intervals $[k(\pi / 8),(k+2)(\pi / 8)]$ with $k=1,3,5$, and $\phi$ in the 8 intervals $[t(\pi / 4),(t+1)(\pi / 4)]$ with $t \in\{0, \ldots, 7\}$. This leaves two classes, surrounding the two poles, corresponding to $\theta \in[0,(\pi / 8)]$ and $\theta \in\left[\frac{7}{8} \pi, \pi\right]$. We train the $\mathrm{CNN}$ with 500 simulated images per class in the training set, and 125 per class in the validation one. The maximum achieved accuracy is $\sim 0.90$. The suboptimality of this result is likely a consequence of framing the problem as a classification task. Indeed, partitioning makes VVBs close to the border of two sectors naturally hard to classify. Training a CNN for the corresponding regression task will potentially improve performance.

Dimensionality reduction.-We now present an alternative approach to classify VVBs from experimental data, leveraging dimensionality reduction (DR). Such algorithms are typically used to obtain efficient representations of large datasets $[73,74]$. This has several advantages, from easing data visualization, to improving the efficiency of classification and regression algorithms, which can be used on the reduced representation of the data. In particular, we employ a linear principal component analysis (PCA) algorithm, which works by representing each datapoint as a vector in some high-dimensional space $\mathbb{R}^{n}$, and finding the directions in such space that capture the maximum amount of information about the dataset [75,81]. The 
rationale for using PCA in this context is that, although experimental images live in extremely high-dimensional spaces (whose dimension is of the order of the number of pixels in the CCD camera), the underlying dimension of the generated VVBs is typically much lower. This means that, although the experimental dataset will a priori seem like a complicated bundle of high-dimensional vectors, the underlying data is actually characterizable by a small number of parameters. Furthermore, the linearity of the mapping preserves the convexity of the VVB space and thus its geometrical structure. We then expect that the new description for expressing the experimental images in the reduced space provides a synthetic description for capturing the features of VVBs encoded in the measurements (the intensities in three polarization bases $\left\{b_{j}\right\}$, cf. Ref. [66]). This resembles a form of unsupervised learning, as we gain useful information about the origin of the images without feeding the algorithm with any knowledge of the underlying process.

As a notable example, we apply these observations to VVBs with $m_{2}=-m_{1}=1$, which can be represented on a sphere in the higher-order Poincaré representation. Indeed, applying PCA to the experimental dataset of Fig. 4(b) reveals that three directions are sufficient to capture most of the information content of the images. Projecting the images along these three principal components, we find that the data are arranged in the form of a three-dimensional (a)

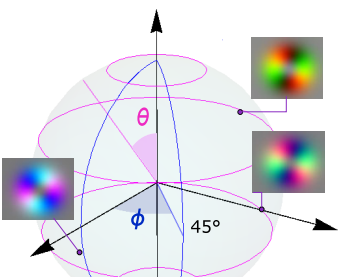

(c)

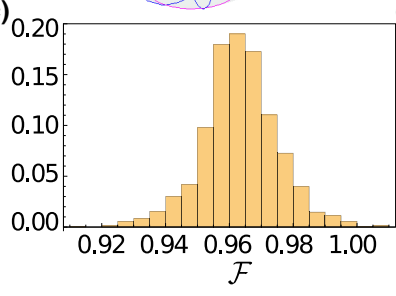

(b)

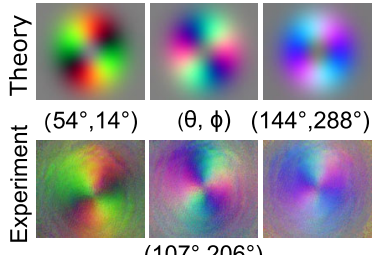

(d) 1.0

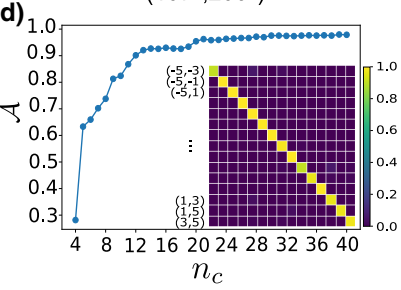

FIG. 4. (a) Higher-order Poincaré sphere for VVBs with $\left|m_{1,2}\right|=1$. Magenta-colored parallels (blue-colored meridians) mark intervals between consecutive values of $\theta(\phi)$. Along a meridian the colors of the pattern vary from the hottest to the coldest one. Along a parallel, the patterns rotate. (b) Comparison between experimental and simulated VVB images for different angles $(\theta, \phi)$. (c) Distribution of fidelities obtained comparing each experimental VVB with its reduced 3D representations given by PCA. (d) Average prediction accuracy $\mathcal{A}$ of a linear SVM classifier, trained and tested after applying linear DR to the data, against the number of reduced dimensions $n_{c}$. For each of the 15 classes (cf. Fig. 3a) in which the experimental dataset was divided, we show in the inset the truth table. sphere embedded in the experimental high-dimensional space. We refer to the Supplemental Material [66] for the distribution of radii of the three-dimensional representation of the images that allows us to retrieve the state's position on the Poincare sphere overcoming the border problem characterizing the previous classification method. Remarkably, this was not obvious from the experimental dataset alone, but was easily revealed using DR. This result highlights the potential of DR to reveal features of the underlying states generating a given experimental dataset in realistic experimental conditions (cf. Refs. [66,67]). Interpreting this reduced three-dimensional representation as a Bloch sphere, we can use PCA to retrieve a complete description of the state generating a given experimental image. To assess the accuracy of such reconstruction, we compute the average fidelity $\mathcal{F}_{\text {avg }}$ between the state generating a given image and the one retrieved from said image via PCA, averaging over many experimental images. The fidelity between two states is here defined in the usual way as $\mathcal{F}(\rho, \sigma) \equiv \operatorname{Tr}|\sqrt{\rho} \sqrt{\sigma}|$. As shown in the histogram of Fig. 4(c), this is found to be $\mathcal{F}_{\text {avg }} \sim 0.96$, with standard deviation $\sim 0.01$, thus showcasing the quality of the reconstruction.

Classification via SVMs.-We now show how the reduced representations provided by PCA can function as a starting point to train a classifier with accuracy comparable with the CNN, while requiring a significantly reduced amount of computational resources. More precisely, we use as classifiers linear support vector machines (SVMs) [76,77]. These supervised learning algorithms categorize data by finding the hyperplane that optimally separates the training dataset in accordance with the corresponding labels.

As done for the $\mathrm{CNN}$, we consider the task of classifying the experimental dataset of VVB states, indexed by $\left(m_{1}, m_{2}\right)$. We train the SVM on the reduced space obtained via PCA, applied to the experimental dataset reported in Fig. 3(a). This significantly improves the efficiency of the classifier, which only has to operate on a compressed representation of the images. This method gives an average accuracy of $\sim 98 \%$ when reducing the dimensionality of the dataset to $40[66,67]$. The SVM was trained on half of the experimental data, with the other half used to test the resulting accuracy. A breakdown of the resulting classification performance is reported in the inset of Fig. 4(d), in which we give the accuracy of the classifier for each class. Finally, we highlight in Fig. 4(d) how the average overall accuracy depends on the dimensionality of the reduced representation. In particular, we find that $\sim 25$ dimensions are already sufficient to get good average accuracies.

Discussion.-We presented a novel approach to classify VVBs leveraging ML techniques. We demonstrated how the use of inference strategies based on CNNs and PCA (enhanced by SVMs) allows us to extract efficiently properties of high-dimensional photonic VVB systems. 
In particular, DR was used to obtain a deeper understanding of the underlying geometrical properties of the experimentally generated states, without requiring prior knowledge about the physics of the generation apparatus. By embedding a variety of ML algorithms into our experimental pipeline, the task of characterizing structured light is made significantly broader in the methods, ranging from supervised to unsupervised learning, and more flexible in the applications, classification and regression tasks. While paving the way to further experimental validations- potentially also in experimental settings that do not rely on optical networks-we believe that numerous tasks of relevance to modern photonics could benefit from introducing similar ML ideas into their characterization protocols. These techniques can prove to be a useful add-on to tasks ranging from the design of automatized approaches to the characterization of experimental platforms and experiments, to the provision of solutions to OAM demultiplexing in the context of classical and quantum communication and, more generally, for the use of structured light in quantum technologies.

We acknowledge support from the ERC Advanced grant PHOSPhOR (Photonics of Spin-Orbit Optical Phenomena; Grant Agreement No. 828978), the EU Collaborative project TEQ (Grant No. 766900), Fondazione Angelo della Riccia, the DfE-SFI Investigator Programme (Grant No. 15/ IA/2864), COST Action CA15220, the Royal Society Wolfson Research Fellowship (RSWFIR3\183013), the Leverhulme Trust Research Project Grant (Grant No. RGP-2018-266).

Note added.-Recently, the authors became aware of a related work [82] that addresses the classification of scalar fields with fractional topological charge.

[1] L. Allen, M. W. Beijersbergen, R. J. C. Spreeuw, and J. P. Woerdman, Phys. Rev. A 45, 8185 (1992).

[2] M. Padgett, J. Courtial, and L. Allen, Phys. Today 57, No. 5, 35 (2004).

[3] M. Erhard, R. Fickler, M. Krenn, and A. Zeilinger, Light Sci. Appl. 7, 17146 (2018).

[4] L. Marrucci, E. Karimi, S. Slussarenko, B. Piccirillo, E. Santamato, E. Nagali, and F. Sciarrino, J. Opt. 13, 064001 (2011).

[5] D. Cozzolino, B. Da Lio, D. Bacco, and L. K. Oxenlwe, Adv. Quantum Technol. 2, 1900038 (2019)..

[6] F. Cardano and L. Marrucci, Nat. Photonics 9, 776 (2015).

[7] H. Rubinsztein-Dunlop et al., J. Opt. 19, 013001 (2017).

[8] A. E. Willner, H. Huang, Y. Yan, Y. Ren, N. Ahmed, G. Xie, C. Bao, L. Li, Y. Cao, Z. Zhao, J. Wang, M. P. J. Lavery, M. Tur, S. Ramachandran, A. F. Molisch, N. Ashrafi, and S. Ashrafi, Adv. Opt. Photonics 7, 66 (2015).

[9] D. Cozzolino, E. Polino, M. Valeri, G. Carvacho, D. Bacco, N. Spagnolo, L. K. Oxenløwe, and F. Sciarrino, Adv. Opt. Photonics 1, 046005 (2019).
[10] R. Fickler, R. Lapkiewicz, W. N. Plick, M. Krenn, C. Schaeff, S. Ramelow, and A. Zeilinger, Science 338, 640 (2012).

[11] V. D’Ambrosio, N. Spagnolo, L. Del Re, S. Slussarenko, Y. Li, L. C. Kwek, L. Marrucci, S. P. Walborn, L. Aolita, and F. Sciarrino, Nat. Commun. 4, 2432 (2013).

[12] K. Goswami, C. Giarmatzi, M. Kewming, F. Costa, C. Branciard, J. Romero, and A. G. White, Phys. Rev. Lett. 121, 090503 (2018).

[13] G. Vallone, V. DAmbrosio, A. Sponselli, S. Slussarenko, L. Marrucci, F. Sciarrino, and P. Villoresi, Phys. Rev. Lett. 113, 060503 (2014).

[14] X.-L. Wang, X.-D. Cai, Z.-E. Su, M.-C. Chen, D. Wu, L. Li, N.-L. Liu, C.-Y. Lu, and J.-W. Pan, Nature (London) 518, 516 (2015).

[15] M. Mirhosseini, O. S. Magaña-Loaiza, M. N. O’Sullivan, B. Rodenburg, M. Malik, M. P. J. Lavery, M. J. Padgett, D. J. Gauthier, and R. W. Boyd, New J. Phys. 17, 033033 (2015).

[16] M. Malik, M. Erhard, M. Huber, M. Krenn, R. Fickler, and A. Zeilinger, Nat. Photonics 10, 248 (2016).

[17] A. Sit, F. Bouchard, R. Fickler, J. Gagnon-Bischoff, H. Larocque, K. Heshami, D. Elser, C. Peuntinger, K. Günthner, B. Heim, C. Marquardt, G. Leuchs, R. W. Boyd, and E. Karimi, Optica 4, 1006 (2017).

[18] D. Cozzolino, D. Bacco, B. Da Lio, K. Ingerslev, Y. Ding, K. Dalgaard, P. Kristensen, M. Galili, K. Rottwitt, S. Ramachandran, and L. K. Oxenløwe, Phys. Rev. Applied 11, 064058 (2019).

[19] P. Zhang, B. H. Liu, R. F. Liu, H. R. Li, F. L. Li, and G. C. Guo, Phys. Rev. A 81, 052322 (2010).

[20] S. K. Goyal, F. S. Roux, A. Forbes, and T. Konrad, Phys. Rev. Lett. 110, 263602 (2013).

[21] F. Cardano, F. Massa, H. Qassim, E. Karimi, S. Slussarenko, D. Paparo, C. de Lisio, F. Sciarrino, E. Santamato, R. W. Boyd, and L. Marrucci, Sci. Adv. 1, e1500087 (2015).

[22] F. Cardano, M. Maffei, F. Massa, B. Piccirillo, C. de Lisio, G. D. Filippis, V. Cataudella, E. Santamato, and L. Marrucci, Nat. Commun. 7, 11439 (2016).

[23] F. Cardano, A. D'Errico, A. Dauphin, M. Maffei, B. Piccirillo, C. de Lisio, G. D. Filippis, V. Cataudella, E. Santamato, L. Marrucci, M. Lewenstein, and P. Massignan, Nat. Commun. 8, 15516 (2017).

[24] L. Innocenti, H. Majury, T. Giordani, N. Spagnolo, F. Sciarrino, M. Paternostro, and A. Ferraro, Phys. Rev. A 96, 062326 (2017).

[25] T. Giordani, E. Polino, S. Emiliani, A. Suprano, L. Innocenti, H. Majury, L. Marrucci, M. Paternostro, A. Ferraro, N. Spagnolo, and F. Sciarrino, Phys. Rev. Lett. 122, 020503 (2019).

[26] J. Leach, M. J. Padgett, S. M. Barnett, S. Franke-Arnold, and J. Courtial, Phys. Rev. Lett. 88, 257901 (2002).

[27] S. Slussarenko, V. D’Ambrosio, B. Piccirillo, L. Marrucci, and E. Santamato, Opt. Express 18, 27205 (2010).

[28] T. Bauer, S. Orlov, U. Peschel, P. Banzer, and G. Leuchs, Nat. Photonics 8, 23 (2014).

[29] G. C. G. Berkhout, M. P. J. Lavery, J. Courtial, M. W. Beijersbergen, and M. J. Padgett, Phys. Rev. Lett. 105, 153601 (2010). 
[30] E. Bolduc, N. Bent, E. Santamato, E. Karimi, and R. W. Boyd, Opt. Lett. 38, 3546 (2013).

[31] M. Malik, M. Mirhosseini, M. P. J. Lavery, M. J. Leach, Jonathan abd Padgett, and R. W. Boyd, Nat. Commun. 5, 3115 (2014).

[32] H. Qassim, F. M. Miatto, J. P. Torres, M. J. Padgett, E. Karimi, and R. W. Boyd, J. Opt. Soc. Am. B 31, A20 (2014).

[33] M. Paris and J. Řeháček, Quantum State Estimation, Lecture Notes in Physics Vol. 649 (Springer, Berlin, 2004).

[34] K. Banaszek, M. Cramer, and D. Gross, New J. Phys. 15, 125020 (2013).

[35] L. Zhenxing, L. Yuanyuan, K. Yougang, L. Yachao, S. Weixing, L. Hailu, and W. Shuangchun, Photonics Res. 5, 15 (2017).

[36] B. Ndagano, I. Nape, M. A. Cox, C. Rosales-Guzman, and A. Forbes, J. Lightwave Technol. 36, 292 (2018).

[37] Y. Chen, J. Gao, Z.-Q. Jiao, K. Sun, W.-G. Shen, L.-F. Qiao, H. Tang, X.-F. Lin, and X.-M. Jin, Phys. Rev. Lett. 121, 233602 (2018).

[38] X. Cai, J. Wang, M. J. Strain, B. Johnson-Morris, J. Zhu, M. Sorel, J. L. O'Brien, M. G. Thompson, and S. Yu, Science 338, 363 (2012).

[39] J. Liu, S.-M. Li, L. Zhu, A.-D. Wang, S. Chen, C. Klitis, C. Du, Q. Mo, M. Sorel, S.-Y. Yu, X.-L. Cai, and J. Wang, Light Sci. Appl. 7, 17148 (2018).

[40] E. Karimi, S. A. Schulz, I. De Leon, H. Qassim, J. Upham, and R.W. Boyd, Light Sci. Appl. 3, e167 (2014).

[41] F. Yue, D. Wen, J. Xin, B. D. Gerardot, J. Li, and X. Chen, ACS Photonics 3, 1558 (2016).

[42] J. Carrasquilla, G. Torlai, R. G. Melko, and L. Aolita, Nat. Mach. Intell. 1, 155 (2019).

[43] T. Giordani, F. Flamini, M. Pompili, N. Viggianiello, N. Spagnolo, A. Crespi, R. Osellame, N. Wiebe, M. Walschaers, A. Buchleitner et al., Nat. Photonics 12, 173 (2018).

[44] R. Santagati, J. Wang, A. A. Gentile, S. Paesani, N. Wiebe, J. R. McClean, S. Morley-Short, P. J. Shadbolt, D. Bonneau, J. W. Silverstone, D. P. Tew, X. Zhou, J. L. O'Brien, and M. G. Thompson, Sci. Adv. 4, eaap9646 (2018).

[45] I. Agresti, N. Viggianiello, F. Flamini, N. Spagnolo, A. Crespi, R. Osellame, N. Wiebe, and F. Sciarrino, Phys. Rev. X 9, 011013 (2019).

[46] A. Lumino, E. Polino, A. S. Rab, G. Milani, N. Spagnolo, N. Wiebe, and F. Sciarrino, Phys. Rev. Applied 10, 044033 (2018).

[47] A. Rocchetto, S. Aaronson, S. Severini, G. Carvacho, D. Poderini, I. Agresti, M. Bentivegna, and F. Sciarrino, Sci. Adv. 5, eaau1946 (2019).

[48] K. T. Butler, D. W. Davies, H. Cartwright, O. Isayev, and A. Walsh, Nature (London) 559, 547 (2018).

[49] C. C. Fischer, K. J. Tibbetts, D. Morgan, and G. Ceder, Nat. Mater. 5, 641 (2006).

[50] A. A. Melnikov, H. P. Nautrup, M. Krenn, V. Dunjko, M. Tiersch, A. Zeilinger, and H. J. Briegel, Proc. Natl. Acad. Sci. U.S.A. 115, 1221 (2018).

[51] J. Wang, S. Paesani, R. Santagati, S. Knauer, A. A. Gentile, N. Wiebe, M. Petruzzella, J. L. OBrien, J. G. Rarity, A. Laing et al., Nat. Phys. 13, 551 (2017).
[52] V. Cimini, I. Gianani, N. Spagnolo, F. Leccese, F. Sciarrino, and M. Barbieri, Phys. Rev. Lett. 123, 230502 (2019).

[53] M. Krenn, R. Fickler, M. Fink, J. Handsteiner, M. Malik, T. Scheidl, R. Ursin, and A. Zeilinger, New J. Phys. 16, 113028 (2014).

[54] M. Krenn, J. Handsteiner, M. Fink, R. Fickler, R. Ursin, M. Malik, and A. Zeilinger, Proc. Natl. Acad. Sci. U.S.A. 113, 13648 (2016).

[55] T. Doster and A. T. Watnik, Appl. Opt. 56, 3386 (2017).

[56] S. R. Park, L. Cattell, J. M. Nichols, A. Watnik, T. Doster, and G. K. Rohde, Opt. Express 26, 4004 (2018).

[57] S. Lohani and R. T. Glasser, Opt. Lett. 43, 2611 (2018).

[58] J. Li, M. Zhang, D. Wang, S. Wu, and Y. Zhan, Opt. Express 26, 10494 (2018).

[59] G. Milione, H. I. Sztul, D. A. Nolan, and R. R. Alfano, Phys. Rev. Lett. 107, 053601 (2011).

[60] F. Cardano, E. Karimi, S. Slussarenko, L. Marrucci, C. de Lisio, and E. Santamato, Appl. Opt. 51, C1 (2012).

[61] L. Marrucci, C. Manzo, and D. Paparo, Phys. Rev. Lett. 96, 163905 (2006).

[62] Y. LeCun, Y. Bengio, and G. Hinton, Nature (London) 521, 436 (2015).

[63] P. Y. Simard, D. Steinkraus, and J. C. Platt, in Proceedings of International Conference on Document Analysis and Recognition (IEEE, New York, 2003), https://ieeexplore .ieee.org/document/1227801.

[64] D. C. Cireşan, U. Meier, J. Masci, L. M. Gambardella, and J. Schmidhuber, in Proceedings of the Twenty-Second International Joint Conference on Artificial IntelligenceVolume Volume Two, IJCAI'11 (AAAI Press, 2011), pp. 1237-1242.

[65] M. Matsugu, K. Mori, Y. Mitari, and Y. Kaneda, Neural Netw. 16, 555 (2003).

[66] See Supplemental Material at http://link.aps.org/ supplemental/10.1103/PhysRevLett.124.160401 which provides further details of the experimental setup and the machine learning techniques, and includes Refs. [22,25,26, 60-62,68-78].

[67] The source code and the dataset for reproducing the results of this work are available at https://github.com/ lucainnocenti/ML-classification-of-VVBs.

[68] F. Chollet et al., Keras," https://keras.io (2015).

[69] M. Abadi et al., TensorFlow: Large-scale machine learning on heterogeneous systems (2015), software available from tensorflow.org.

[70] S. Ruder, arXiv:1609.04747.

[71] E. Karimi, G. Zito, B. Piccirillo, L. Marrucci, and E. Santamato, Opt. Lett. 32, 3053 (2007).

[72] E. Karimi, B. Piccirillo, L. Marrucci, and E. Santamato, Opt. Lett. 34, 1225 (2009).

[73] P. Cunningham, in Machine Learning Techniques for Multimedia (Springer, Berlin, 2008), pp. 91-112.

[74] I. K. Fodor, A survey of dimension reduction techniques, Technical Report, Lawrence Livermore National Lab., CA, US, 2002.

[75] I. T. Jolliffe and J. Cadima, Phil. Trans. R. Soc. A 374, 20150202 (2016).

[76] M. A. Hearst, S. T. Dumais, E. Osuna, J. Platt, and B. Scholkopf, IEEE Intell. Syst. Appl. 13, 18 (1998). 
[77] N. Cristianini and J. Shawe-Taylor, An Introduction to Support Vector Machines and Other Kernel-based Learning Methods (Cambridge University Press, Cambridge, England, 2000).

[78] M. Rafayelyan, T. Gertus, and E. Brasselet, Appl. Phys. Lett. 110, 261108 (2017).

[79] W. Shu, Y. Liu, Y. Ke, X. Ling, Z. Liu, B. Huang, H. Luo, and X. Yin, Opt. Express 24, 21177 (2016).
[80] G. Vallone, A. Sponselli, V. D’Ambrosio, L. Marrucci, F. Sciarrino, and P. Villoresi, Opt. Express 24, 16390 (2016).

[81] I. Jolliffe, Principal Component Analysis (Springer, Berlin, Heidelberg, 2011).

[82] Z. Liu, S. Yan, H. Liu, and X. Chen, Phys. Rev. Lett. 123, 183902 (2019). 